Nonlinear Processes in Geophysics, 12, 767-774, 2005

SRef-ID: $1607-7946 / \mathrm{npg} / 2005-12-767$

European Geosciences Union

(c) 2005 Author(s). This work is licensed

under a Creative Commons License.

\title{
Scaling collapse and structure functions: identifying self-affinity in finite length time series
}

\author{
S. C. Chapman ${ }^{1}$, B. Hnat ${ }^{1}$, G. Rowlands ${ }^{1}$, and N. W. Watkins ${ }^{2}$ \\ ${ }^{1}$ Space and Astrophysics, University of Warwick, UK \\ ${ }^{2}$ British Antarctic Survey (NERC), Cambridge, UK
}

Received: 23 March 2005 - Revised: 1 June 2005 - Accepted: 12 July 2005 - Published: 3 August 2005

\begin{abstract}
Empirical determination of the scaling properties and exponents of time series presents a formidable challenge in testing, and developing, a theoretical understanding of turbulence and other out-of-equilibrium phenomena. We discuss the special case of self affine time series in the context of a stochastic process. We highlight two complementary approaches to the differenced variable of the data: i) attempting a scaling collapse of the Probability Density Functions which should then be well described by the solution of the corresponding Fokker-Planck equation and ii) using structure functions to determine the scaling properties of the higher order moments. We consider a method of conditioning that recovers the underlying self affine scaling in a finite length time series, and illustrate it using a Lévy flight.
\end{abstract}

\section{Introduction}

Theories of turbulence as applied to single point measurements in a flow concern the scaling properties, in a statistical sense, of differenced time series, where the Taylor hypothesis is invoked so that the difference between measurements at some time $t$ and a later time $t+\tau$ acts as a proxy for the difference between measurements made at two points in the fluid separated by length scale $L$. Studies of scaling in solar wind turbulence have focused on the power spectra and the structure functions (see e.g. Tu and Marsch, 1995; Horbury and Balogh, 1997) and, more recently, the Probability Density Function (PDF) (see Hnat et al., 2002, 2003b).

The statistical scaling properties of time series can in general, however, be considered in a similar manner. There is a considerable literature concerning scaling in auroral region magnetometers and in geomagnetic indices (such as Tsurutani et al., 1990; Takalo et al., 1993; Consolini et al., 1996; Vörös et al., 1998; Uritsky and Pudovkin, 1998; Watkins et al., 2001; Kovács et al., 2001). This is motivated in part

Correspondence to: S. C. Chapman

(sandrac@astro.warwick.ac.uk) by attempts to understand the driven magnetospheric system from the perspective of scaling due to intrinsic processes (see e.g. Chapman and Watkins, 2001, and references therein) and their relationship to that of the turbulent solar wind driver. This necessitates quantitative "comparative" studies of scaling in time series (e.g. Takalo and Timonen, 1998; Freeman et al., 2000; Uritsky et al., 2001; Vörös et al., 2002; Hnat et al., 2003a). Such studies can to some extent fruitfully consider the low order moments, whereas a particular difficulty for comparison of observations with models of turbulence is that the intermittency parameter in turbulence $\tau(2)$ is determined by the 6th order structure function (Frisch, 1995).

More recently, studies have focussed on the scaling properties and functional form of the PDFs of the differenced time series (see e.g. Consolini and De Michelis, 1998; SorrisoValvo et al., 2001; Weigel and Baker, 2003a; Stepanova et al., 2003). This leads to a Fokker-Planck model in the case of self-similarity (Hnat et al., 2003b, 2005).

In this paper we describe an approach to modelling such scaling data which exploits the data's self-affine property by applying the idea of coarse graining the data (Wilson, 1979; Sornette, 2000), here in the time domain. This coarsegraining can be achieved empirically, from the data, by a scaling collapse procedure (e.g. Hnat et al., 2003b, 2005) (Sect. 2), and, then having experimentally determined the scaling exponent, we can take the approach one stage further and seek to describe the data by means of a particular case of a generalised Fokker-Planck equation (GFPE, Sect. 5). We stress here that the GFPE is here, as elsewhere (e.g. Sornette, 2000), applied to a much more general class of problem than the strictly equilibrium physics for which the original FPE was obtained. The GFPE represents an alternative to the fractional Fokker-Planck equation (e.g. Zaslavsky, 1995) which is also applicable in such non-equilibrium cases.

The critical steps in this process are then (i) establishing whether a given dataset is self affine and (ii) determining the scaling exponent. We highlight two important issues that arise in the analysis of physical datasets here. 
The first of these is that SDE (Stochastic Differential Equations) models for the data, and indeed, coarse graining, deal with the properties of an arbitrarily large dataset. We use a well understood example of a self affine time series, that of ordinary Lévy motion (Sect. 3), to show how conditioning of the data is needed to recover the known scaling of an arbitrarily large time series from one finite length. We then use an example of a naturally occurring time series, that of the $A E$ geomagnetic index, shown previously to exhibit self affine scaling over a range of timescales, to highlight the effectiveness, and the limitations, of this technique.

The second of these is that knowledge of the scaling properties of (in principle all) the non zero moments is needed to capture the scaling properties of a time series. We again use the $A E$ time series to illustrate this point by constructing a fractional Brownian motion $\mathrm{fBm}$ with the same second moment, but with a very different PDF.

\section{Self affine time series: concepts}

From a time series $x(t)$ sampled at times $t_{k}$, that is at evenly spaced intervals $\Delta=t_{k}-t_{k-1}$ we can construct a differenced time series with respect to the time increment $\tau=s \Delta$ :

$y(t, \tau)=x(t+\tau)-x(t)$

so that

$x(t+\tau)=x(t)+y(t, \tau)$.

If we consider $N$ successive values determined at intervals of $\Delta$, that is, $y\left(t_{1}, \Delta\right) \ldots y\left(t_{k}, \Delta\right) \ldots y\left(t_{N}, \Delta\right)$, their sum gives:

$x(t)=\sum_{1}^{N} y\left(t_{k}, \Delta\right)+x_{0}$,

where $x_{0}=x(t-N \Delta)$. As $N \rightarrow \infty$ the sum (Eq. 3) of the $y$ tends to the original time series $x(t)$.

We will make two assumptions: i) that the $y(t, \tau)$ is a stochastic variable so that Eq. (2) can be read as a random walk and ii) that the $y$ are scaling with $\tau$ (to be defined next).

By summing adjacent pairs in the sequence, for example:

$y^{(1)}\left(t_{1}, 2 \Delta\right)=y\left(t_{1}, \Delta\right)+y\left(t_{2}, \Delta\right)$

one can coarsegrain (or decimate) the time series in $\tau$. This operation gives the $x(t)$ as a random walk of $N / 2$ values of $y$ determined at intervals of $\tau=2 \Delta$. We can successively coarsegrain the sequence an arbitrary number of times:

$$
\begin{aligned}
x(t)= & y\left(t_{1}, \Delta\right)+y\left(t_{2}, \Delta\right)+\cdots+y\left(t_{k}, \Delta\right)+y\left(t_{k+1}, \Delta\right)(5) \\
& +\cdots+y\left(t_{N}, \Delta\right) \\
= & y^{(1)}\left(t_{1}, 2 \Delta\right)+\cdots+y^{(1)}\left(t_{k}, 2 \Delta\right) \\
& +\cdots+y^{(1)}\left(t_{N / 2}, 2 \Delta\right) \\
= & y^{(n)}\left(t_{1}, 2^{n} \Delta\right)+\cdots+y^{(n)}\left(t_{k}, 2^{n} \Delta\right) \\
& +\cdots+y^{(n)}\left(t_{N / 2^{n}}, 2^{n} \Delta\right),
\end{aligned}
$$

where this procedure is understood in the renormalization sense in that both $N$ and $n$ can be taken arbitrarily large, so that a time series of arbitrarily large length is considered. This procedure can apply to a finite sized physical system of interest provided that that system supports a large range of spatio-temporal scales (the smallest being $\Delta$, the largest, $2^{n} \Delta, n$ large), an example of this is the inertial range in fluid turbulence.

We now consider a "self affine" scaling with exponent $\alpha$ :

$y^{\prime}=2^{\alpha} y, \quad \tau^{\prime}=2 \tau$,

so that

$y^{(n)}=2^{n \alpha} y, \quad \tau=2^{n} \Delta$.

For arbitrary $\tau$ we can normalize $(\tau \equiv \tau / \Delta)$ and write

$y^{\prime}(t, \tau)=\tau^{\alpha} y(t, \Delta)$.

Now if the $y$ is a stochastic variable with self affine scaling in $\tau$, there exists a "self similar" PDF which is unchanged under the transformation given by Eq. (8):

$P\left(y^{\prime} \tau^{-\alpha}\right) \tau^{-\alpha}=P(y)$.

Importantly, the $y^{\prime} s$ are not necessarily Gaussian distributed stochastic variables, but do possess self similarity as embodied by Eq. (9).

This property is shared by the ( $\alpha$-stable) Lévy flights (Shlesinger et al., 1995) for $N \rightarrow \infty$. The special case where the $y^{\prime} s$ are both independent, identically distributed (iid) and have finite variance corresponds to a Brownian random walk. One can show directly from the above renormalization (see for example Sornette, 2000) that the Brownian case is just the Central Limit Theorem with $\alpha=1 / 2$ and Gaussian $P(y)$. Here, we consider time series which possess the properties (Eqs. 8 and 9), which may have $\alpha \neq 1 / 2$ and which are time stationary solutions of a Fokker-Planck equation.

An important corollary of Eq. (9) is of the scaling of the structure functions (and moments). The $p$ th moment can be written as:

$m_{p}=<y^{p}>=\int_{-\infty}^{\infty} P(y) y^{p} d y=\tau^{p \alpha} \int_{-\infty}^{\infty} P\left(y^{\prime}\right) y^{\prime p} d y^{\prime}$

so that

$m_{p} \sim \tau^{p \alpha}$

via Eq. (9). The scaling of any of the non zero moments of a self affine time series is thus sufficient to determine the exponent. Importantly, all the non zero moments will share this same scaling. This can also be appreciated directly by writing the PDF as an expansion in the moments. If we define the Fourier transform of the PDF $P(z)$ of a given time series $z(t)$ by:

$\hat{P}(k)=\int_{-\infty}^{\infty} e^{i k z} P(z) d z$

then it is readily shown that the $p$ th moment is given by:

$m_{p}=\left.(-i)^{p} \frac{d^{p} \hat{P}(k)}{d k^{p}}\right|_{k=0}$ 
where $d^{p} / d k^{p}$ denotes the $p$ th derivative with respect to $k$. From this it follows that the PDF can be expressed as an expansion in the moments:

$\hat{P}(k)=\sum_{p=0}^{\infty} \frac{m_{p}}{p !}(i k)^{p}$.

Hence the PDF is defined by knowledge of "all" the non zero moments.

\section{Testing for self affine scaling}

3.1 Extracting the scaling of a surrogate, a finite length Lévy flight

We now discuss methods for testing for the property (Eq. 9) and measuring the exponent $\alpha$ for a given finite length time series. For the purpose of illustration we consider a Lévy flight of index $\mu=1.8$ which is generated from iid random deviates by the following algorithm for the increments (the $y^{\prime} s$, see Siegert and Friedrich, 2004, for details):

$f_{\mu}=\frac{\sin (\mu r)}{(\cos (r))^{\frac{1}{\mu}}}\left(\frac{\cos [(1-\mu) r]}{v}\right)^{\frac{(1-\mu)}{\mu}}$,

where $r$ is a uniformly distributed random variable in the range $[-\pi / 2, \pi / 2]$ and $v$ is an exponentially distributed random variable with mean 1 which is independent of $r$. The scaling exponent $\alpha$ from Eqs. (8) and (9) is then related to the Lévy index, $\mu$, by $\alpha=1 / \mu$.

One can first consider directly attempting a scaling collapse in the sense of Eq. (9), of the PDF of differences obtained over a wide range of $\tau$ (see Mantegna and Stanley, 1995; Hnat et al., 2003a,b, for example). This corresponds to a renormalization of the data as discussed above. We first determine the scaling exponent $\alpha$ from one or more of the moments via Eq. (11) or an estimate thereof. In a finite length time series, one would ideally use the scaling of the peak $P(y=0, \tau)$ (that is, the $p=-1$ moment) with $\tau$ as this is better resolved statistically than the higher order moments. In practice however the time series $y(t, \tau)$, formed from the differences of a measured quantity, can as $y \rightarrow 0$ be dominated by observational uncertainties.

Figure 1 shows the scaling collapse (Eq. 9) applied to a numerically generated Lévy flight (Eq. 15) of $10^{6}$ increments. The curves correspond to differences at values of $\tau=m \Delta$ with $m=[6,10,16,26,42]$. Error bars denote an estimate of the expected fluctuation per bin of this histogram based on Gaussian statistics (a more sophisticated method for estimating these for the Lévy case may be found in Siegert and Friedrich, 2004). We see that scaling collapse can be verified to the precision with which the PDF is locally determined statistically. The exponent $\alpha=0.544$ used to achieve the scaling collapse in Fig. 1 was determined empirically directly from an analysis of this finite length time series based on the structure functions discussed below.

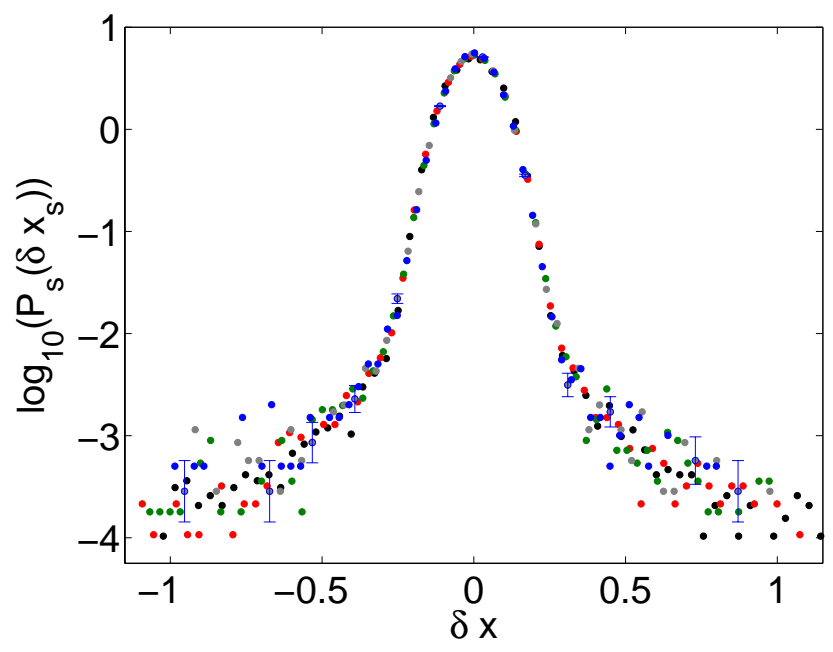

Fig. 1. Scaling collapse of the PDF of an $\mu=1.8$ Lévy flight.

As discussed above, the scaling exponent $\alpha$ that successfully collapses the PDF of different $\tau$ should emerge from the scaling of the moments. This is often obtained via the generalized structure functions (see e.g. Tu and Marsch, 1995; Horbury and Balogh, 1997; Hnat et al., 2003a, 2005, for examples)

$S_{p}(\tau)=<|y(t, \tau)|^{p}>\propto \tau^{\zeta(p)}$,

where for self affine $y(t)$, we have $\zeta(p)=p \alpha$ (for a multifractal, $\zeta(p)$ is approximately quadratic in $p)$. From Eq. (11) the moments will in principle share this scaling provided that the moment is both finite and non- zero (however in a noisy signals a moment that should vanish will be dominated by the noise). In principle we can obtain $\alpha$ from the slopes of $\log$ - $\log$ plots of the $S_{p}$ versus $\tau$ for any $p$; in practice this is severely limited by the finite length of the dataset.

The $\zeta(p)$ for the above Lévy flight obtained via Eq. (16) are shown plotted versus $p$ in Fig. 2. On such a plot we would expect from Fig. 1 a straight line $\zeta(p) \sim p \alpha$ but we see here the well known result (see for example Chechkin and Gonchar, 2000; Nakao, 2000) that for the surrogate, the Lévy time series of finite length, there is a turnover in scaling above $p=\mu$ which is spurious in the sense that it does not reflect the exponent $\mu$ of the infinite length time series.

One way to resolve this apparent bifractal scaling is to note that in a finite length time series the PDF does not have sufficient statistical resolution in the tails. Infrequently occurring large events in the tails will tend to dominate the higher order moments. We now eliminate those large events that are poorly represented statistically without distorting the scaling properties of the time series. For a self affine time series an estimate of the structure functions is:

$S_{p}^{C}=\int_{-A}^{A}|y|^{p} P(y, \tau) d y \approx<|y|^{p}>$

where the limit on the integral is proportional to the standard deviation $\sigma$ so that $A=Q \sigma(\tau)$, with some $Q$ constant. Now 


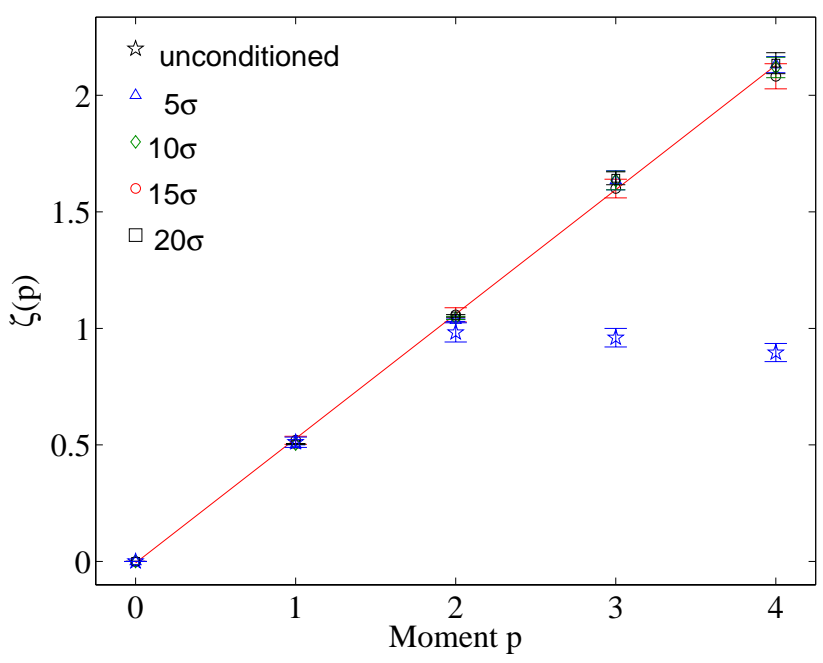

Fig. 2. The effect of conditioning a Lévy flight. $\star$ are the $\zeta(p)$ obtained from the raw time series, all other symbols refer to conditioned time series for different values of $Q$ (see text). The conditioned results yield a scaling exponent $\alpha=0.544$ which corresponds to a Lévy index of $\mu=1.84$.

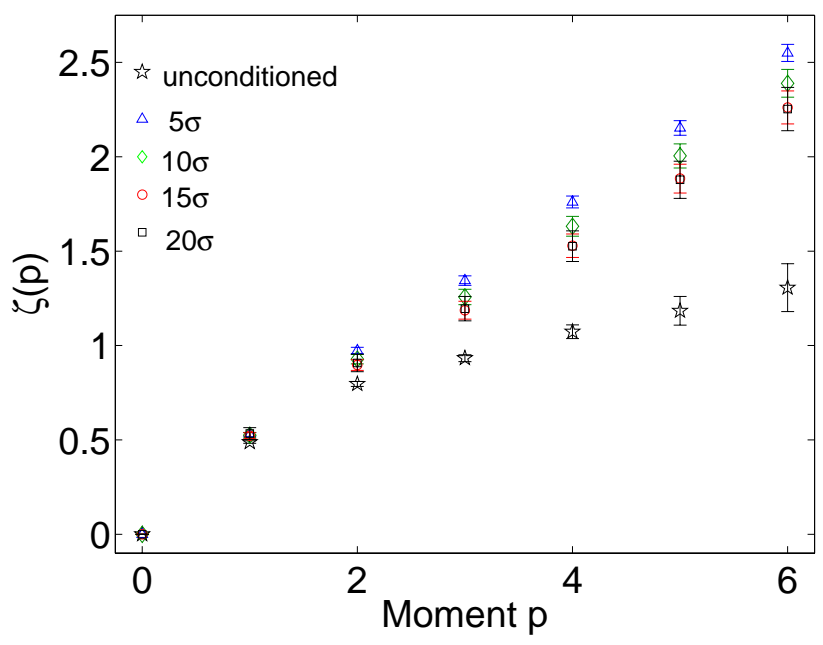

Fig. 3. Scaling exponents $\zeta(p)$ versus $p$ for the $A E$ index, shown in the same format as Fig. 2.

$\sigma(\tau) \sim \sqrt{<y^{2}>} \sim \tau^{\alpha}$ shares the same self affine scaling with $\tau$ as the original time series $y(t, \tau)$, so that if $S_{p} \sim \tau^{p \alpha}$ under Eq. (9) then, importantly, $S_{p}^{C} \sim \tau^{p \alpha}$ also. Provided that $Q$ can be chosen sufficiently large to capture the dynamic range of $y$, and provided that $P(y)$ is symmetric, Eq. (17) will provide a good estimate of $\alpha$. This is demonstrated in Fig. 2 where we also show the $\zeta(p)$ obtained from Eq. (17).

One can thus see that once a conditioning threshold is applied, the self affine scaling of the Lévy flight is recovered and the value of the scaling exponent is insensitive to the value of $Q$ chosen (for $Q$ sufficiently large). We obtain the value of $\alpha=0.544$ used for the scaling collapse in Fig. 1 once conditioning is applied, giving an estimate of $\mu=1.84$, consistent with the index used to generate the synthetic Lévy

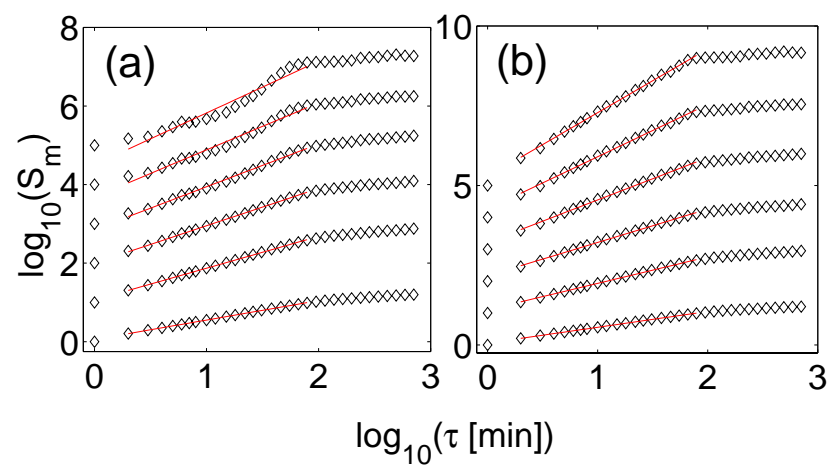

Fig. 4. Structure functions of the $A E$ index estimated for orders $p=[1,6]$ by Eq. (16) (a) and by Eq. (17) (b).

flight (Eq. 15). Similar results for a surrogate Lévy dataset have been obtained by M. Parkinson (private communication, 2004).

An analogous procedure to Eq. (17) can also be realized by means of a truncated wavelet expansion of the data (see for example Kovács et al., 2001; Mangeney et al., 2001).

In Eq. (17) we assumed self affine scaling in choosing the functional form of the limits of the integral. In a given time series the scaling may not be known a priori. If for example the time series were multifractal $(\zeta(p)$ quadratic in $p)$ we would obtain from Eq. (17) a $\zeta(p)$ which varied systematically with $Q$. In practice, several other factors may also be present in a time series which may additionally reduce the accuracy of the approximation of Eq. 17).

3.2 Extracting the scaling of a "natural" example, the $A E$ time series

To illustrate the above, we consider an interval of the $A E$ index shown previously to exhibit weakly multifractal scaling (Hnat et al., 2005). The scaling index is not within the Lévy range and thus it has been modelled with a GFPE rather than a Lévy walk (Hnat et al., 2005).

The PDF of differenced $A E$ is asymmetric (Hnat et al., 2003a), and the scaling in $\tau$ is broken as we approach the characteristic substorm timescale of 1-2h. Remnants of the substorm signature will be present in the time series on timescales shorter than this. The behaviour of the peak of the PDF $(P(y \rightarrow 0))$ will also be dominated by uncertainties in the determination of the signal rather than its scaling properties.

Figure 3 shows a plot of $\zeta(p)$ versus $p$ for the $A E$ time series in the same format as Fig. 2 for the interval January 1978 to July 1979 comprising $7.5 \times 10^{5}$ samples. Plots of the structure functions used to construct Fig. 3 are shown in Fig. 4. The error bars on Fig. 3 are those of the best fit straight lines to Fig. 4 rather than the possible range of straight line fits and as such are a minimal error estimate.

We plot in Fig. 4a the raw result, that is Eq. (16) and in Fig. 4b the conditioned approximation (Eq. 17) with $Q=20$, the latter corresponding to the removal of less than $1 \%$ of 


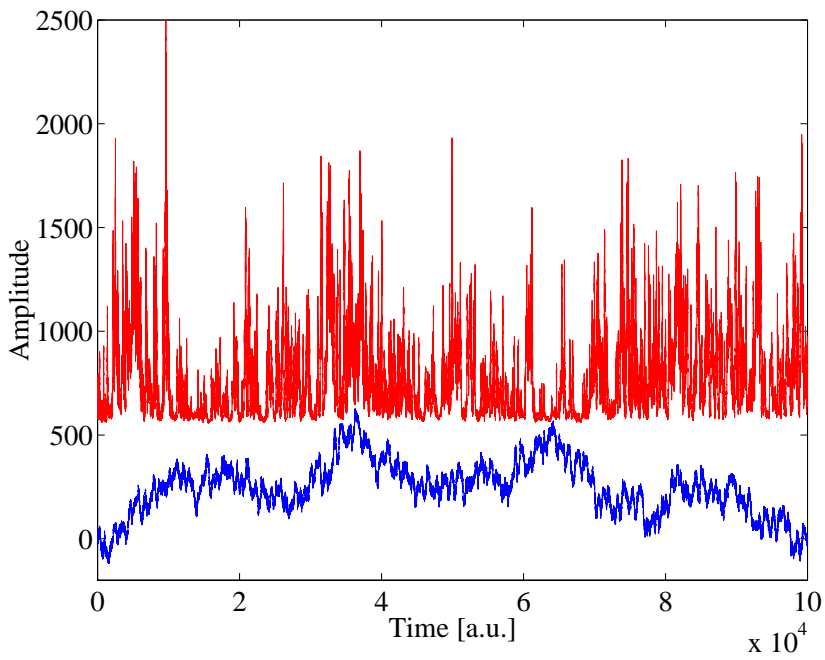

Fig. 5. A $\sim 1.5$ year interval of $A E$ data (upper trace) is shown alongside a surrogate $\mathrm{fBm}$ time series (lower trace) with the same second moment. The traces have been displaced for clarity.

the data. From Fig. 4 we see that no clear scaling emerges beyond the third order $p=3$ until approximation (Eq. 17) is made. Clearly, if scaling is present, the $\zeta(p)$ obtained from the raw structure functions (Fig. 4a) are not a good estimate. Once the data is conditioned, we find that $Q=[10,20]$ give almost identical estimates of $\zeta(p)$ which are weakly multifractal. For $Q=5$ the $\zeta(p)$ are shifted slightly toward self similar scaling. The closeness of the conditioned results for the range $Q=[5,20]$, and their clear separation from the raw result, suggests that these are a reasonable approximate measure of the scaling properties of the time series. This procedure can be used to make quantitative comparisons between time series to this precision. Given the caveats above however, we cannot use this procedure to distinguish whether the time series is self affine or weakly multifractal, but can distinguish strong multifractality.

\section{Low order moments and non uniqueness: comparison with a fractional Brownian surrogate.}

Equation (14) expresses the PDF as an expansion in the moments to all orders. It follows that distinct time series can share the first few moments and therefore if scaling, may also share the same Hurst exponent and corresponding exponent of the power law power spectrum. Having estimated the scaling exponent of the $A E$ index as above we can construct a time series with the same second moment from a fractional Brownian motion to illustrate this.

The fractional Brownian walk was generated using the method described in Appendix 3 of (Peters, 1996). The algorithm takes a series of Gaussian random numbers and approximates a finite correlation time by weighting past values according to a power law function. In our case 1024 Gaussian samples were used to create each increment of fractional

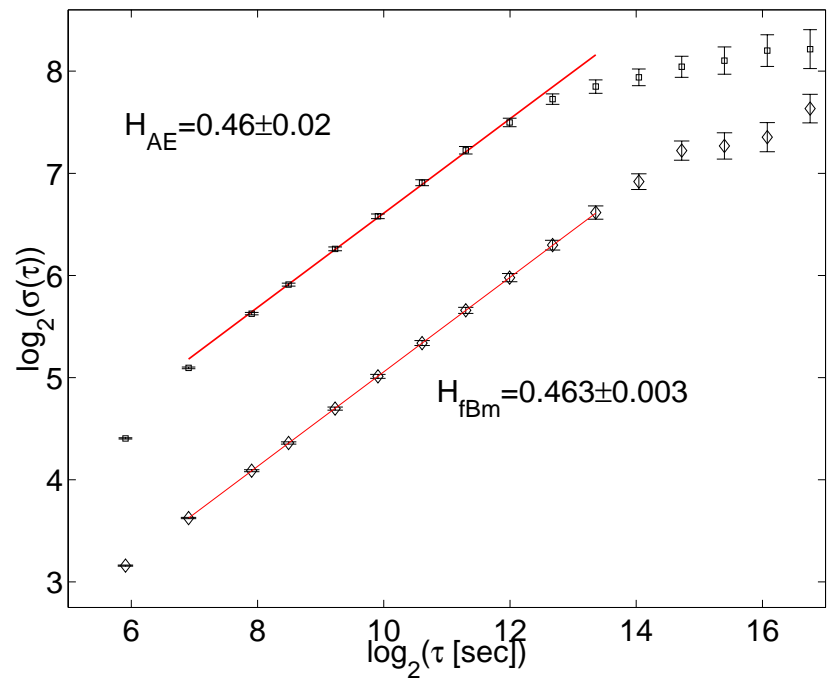

Fig. 6. Standard deviation of the time series differenced on timescale $\tau$ plotted versus $\tau$ for an interval of $A E$ index data (see text) and an fBm time series constructed with the same second moment. The traces have been displaced for clarity.

walk. The resulting time series is comprised of $7.5 \times 10^{5}$ increments.

Figure 5 shows the two time series, (i) the interval of $A E$ analyzed above, and (ii) the $\mathrm{fBm}$ surrogate. The standard deviation versus $\tau$ for the two time series is shown in Fig. 6 . The power spectrum of $A E$ (the raw, rather than the differenced variable)(c.f. Tsurutani et al., 1990; Takalo et al., 1993), along with the $\sigma(\tau)$ and the structure functions, show a characteristic break on timescales corresponding to $1-2 \mathrm{~h}$. On times shorter than this, we can obtain a scaling collapse of the PDF (see Hnat et al., 2003a, 2005). Fluctuations on these timescales share the same second moment as the fBm. In Fig. 7 we compare the PDF of these fluctuations and we see that these are very distinct; $\mathrm{fBm}$ is defined as having Gaussian increments (Mandelbrot, 2002) and this is revealed by the PDF whereas the $A E$ increments are non-Gaussian.

This is an illustration of the fact that the scaling in $A E$ over this region is not necessarily due to time correlation, the "Joseph effect" for which Mandelbrot constructed fractional Brownian motion as a model. Indeed $A E$ has almost uncorrelated differences at high frequencies, as indicated by its nearly Brownian $f^{-2}$ power spectrum (Tsurutani et al., 1990). Rather the scaling is synonymous with the heavy tailed PDF ("Noah effect") for which Mandelbrot (2002) earlier introduced a Lévy model in economics.

Finally, we plot in Fig. 8 the $\zeta(p)$ versus $p$ obtained from the structure function estimate (Eq. 17) with $Q=10$ for both time series. We see from the plot that both time series are self affine and to within the uncertainty of the finite length time series, both share values of $\zeta(p)$ for the lowest orders in $p$. However the higher order structure functions reveal the distinct scaling of the two time series. 


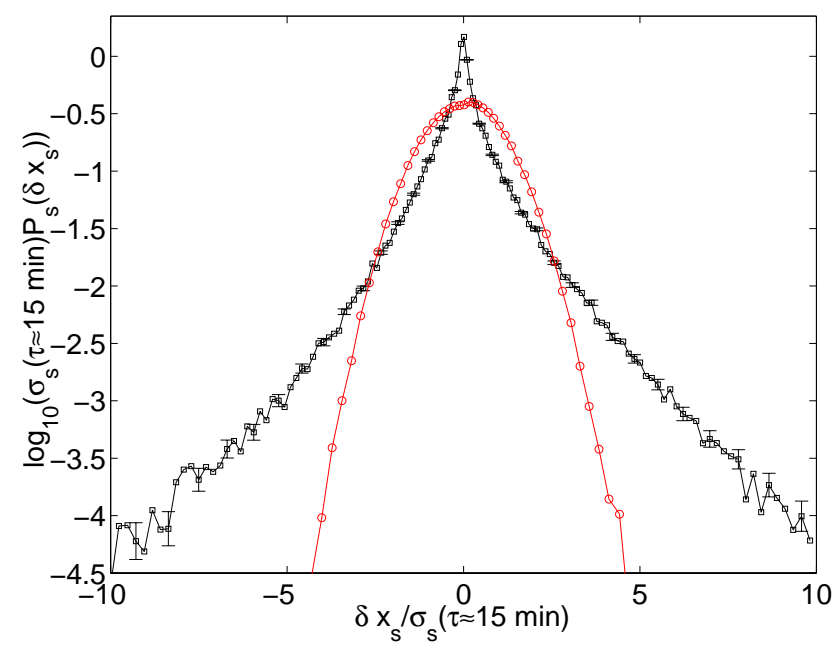

Fig. 7. PDF of the time series of $A E$, differenced on timescales less than one hour ( $\square$ ). The PDF of an fBm with the same second moment is shown for comparison (o).

\section{Fokker-Planck model}

For completeness we now outline how the exponent $\alpha$ of a self affine time series leads to the functional form of $P(y)$ via a Fokker- Planck model of the stochastic process $x(t)$. Here we will consider an approach where scaling is achieved via transport coefficients that are functions of the differenced variable $y(t)$. An alternative approach is via fractional derivatives for the dependent $(y)$ coordinate (see e.g. Schertzer et al., 2001; Shlesinger et al., 1995). These are in principle equivalent (see e.g. Yannacopoulos and Rowlands, 1997).

We begin with a general form of the Fokker-Planck equation which can be written (Gardiner, 1986):

$\frac{\partial P}{\partial \tau}=\nabla_{y}\left(A(y) P+B(y) \nabla_{y} P\right)$,

where $P \equiv P(y, \tau)$ is a PDF for the differenced quantity $y$ that varies with time $\tau, A(y)$ is the friction coefficient and $B(y)$ is related to a diffusion coefficient which we allow to vary with $y$. If we now impose the condition that solutions of Eq. (18) are invariant under the scaling given by Eq. (9), then it is found that both $A(y)$ and $B(y)$ must have the form of power law dependence on $y$. Then as shown in (Hnat et al., 2003b), Eq. (18) takes the form:

$\frac{\partial P}{\partial \tau}=\frac{\partial}{\partial y}\left[y|y|^{-1 / \alpha}\left(a_{0} P+b_{0} y \frac{\partial P}{\partial y}\right)\right]$,

where $a_{0}$ and $b_{0}$ are constants, $\alpha$ is the scaling index derived from the data and $P(y), y$ are unscaled PDF and fluctuations respectively, and where here we have explicitly insisted that the diffusion coefficient $B(y)>0$. Importantly, in a physical system the scaling behaviour (Eq. 9) is expected to be strongly modified as $y \rightarrow 0$, that is, at the peak of the PDF $P(y)$ since for a sufficiently small difference between

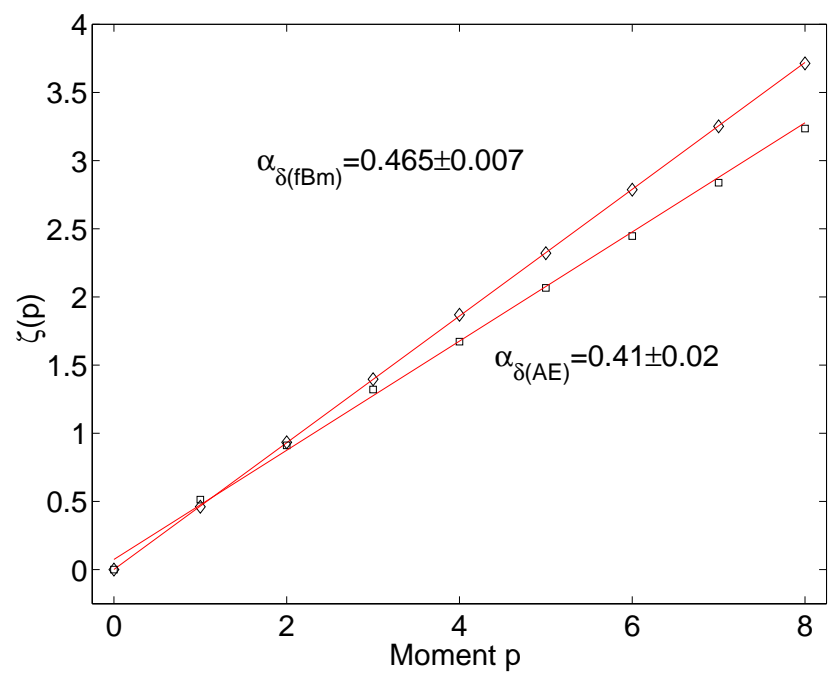

Fig. 8. Structure functions obtained by conditioning at $10 \sigma$ for an interval of the $A E$ index, and for a $\mathrm{fBm}$ constructed to share the same second moment.

two measurements $x(t), y(t, \tau)=x(t+\tau)-x(t)$ will be dominated by the uncertainties in those measurements.

Written in this form Eq. (19) immediately allows us to identity $B(y) \propto y^{2}|y|^{-1 / \alpha}$ and $A(y) \propto y|y|^{-1 / \alpha}$. Solutions to Eq. (19) exist which are functions of $y_{s}=y \tau^{-\alpha}$ only which correspond to stationary solutions with respect to $\tau$. We obtain these by the change of variables $\left(P, y, \tau \rightarrow P_{s}, y_{s}\right)$ of Eq. (19):

$\frac{b_{0}}{a_{0}} y_{s} \frac{d P_{s}}{d y_{s}}+P_{s}+\frac{\alpha}{a_{0}}\left|y_{s}\right|^{\frac{1}{\alpha}} P_{s}=\frac{C\left|y_{s}\right|^{\frac{1}{\alpha}}}{y_{s}}$.

This differential Eq. (20) can be solved analytically with a general solution of the form:

$P_{s}\left(y_{s}\right)=\frac{a_{0}}{b_{0}} \frac{C}{\left|y_{s}\right|_{0} / b_{0}} \exp \left(-\frac{\alpha^{2}}{b_{0}}\left|y_{s}\right|^{1 / \alpha}\right)$
$\times \int_{0}^{y_{s}} \frac{\left|y_{s}^{\prime}\right|^{\frac{a_{0}}{b_{0}}} \exp \left(\frac{\alpha^{2}}{b_{0}}\left|y_{s}^{\prime}\right|^{1 / \alpha}\right)}{\left|y_{s}^{\prime}\right|^{2-\frac{1}{\alpha}}} d\left(y_{s}^{\prime}\right)+k_{0} H\left(y_{s}\right)$,

where $k_{0}$ is a constant and $H\left(y_{s}\right)$ is the homogeneous solution:

$H\left(\delta x_{s}\right)=\frac{1}{\left|y_{s}\right|^{a_{0} / b_{0}}} \exp \left(-\frac{\alpha^{2}}{b_{0}}\left|y_{s}\right|^{1 / \alpha}\right)$.

Power law scaling for arbitrary $y$ leads to singular behaviour of this solution at $y \rightarrow 0$. We do not however expect this to describe a physical system as $y \rightarrow 0$ as discussed above. For Eq. (21) to describe a PDF we require that its integral is finite. We can discuss this by considering the behaviour close to the singularity:

$\lim _{y_{s} \rightarrow 0} P\left(y_{s}\right) \simeq \frac{a_{0}}{b_{0}} \frac{C}{\left|y_{s}\right|^{\frac{a_{0}}{b_{0}}}} \int_{0}^{y_{s}} \frac{\left|y_{s}^{\prime}\right|^{\frac{a_{0}}{b_{0}}} d y_{s}^{\prime}}{y_{s}^{\prime 2-\frac{1}{\alpha}}}+\frac{k_{0}}{\left|y_{s}\right|^{a_{0}}}=$ 


$$
C+\frac{k_{0}}{\left|y_{s}\right|^{\frac{a_{0}}{b_{0}}}} \text {. }
$$

The integral of (23) is finite for $0 \leq a_{0} / b_{0}<1$ and $0<\alpha \leq 1 / 2$ (a subdiffusive process) so that within this range the integral of (21) will be finite also as required. Outside of this range it can only be considered as an asymptotic solution. However, we can consider the generalization $y \rightarrow y+\epsilon$ in the above, where $\epsilon$ is a constant of magnitude that is small compared to, say, the values of $\sigma(\tau)$ for the physical system under study. This eliminates the singular behaviour and corresponds (for $y$ small) to the addition of low amplitude Gaussian noise as can be seen from the form of the corresponding Langevin equation (Eq. 24) below. Physically this corresponds to a simple model for the statistical behaviour of the observational uncertainties in the data which may dominate as the differenced quantity $y \rightarrow 0$.

Expression (21) is then a family of solutions for the PDF of self affine time series. This provides a method to test for self affinity that does not directly rely on determining the scaling exponents to high order from the structure functions. Having determined the exponent $\alpha$ from the scaling of a low order moment (say, the standard deviation) one can then perform a scaling collapse on the PDF; this should then also be described by the corresponding solution of Eq. (21) (see Hnat et al., 2003b, 2005, for example).

It is well known that a Fokker Planck equation is simply related to a Langevin equation (see e.g. Gardiner, 1986). A nonlinear Langevin equation of the form

$\frac{d y}{d t}=\beta(y)+\gamma(y) \xi(t)$

where $\beta(y)$ is a $y$-dependent force term and $\gamma(y)$ is a $y$ dependent noise strength, can be shown (Hnat et al., 2003b) to correspond to Eq. (18) and in that sense to describe the time series. In Eq. (24) the random variable $\xi(t)$ is assumed to be $\delta$-correlated, i.e.

$$
<\xi(t) \xi(t+\tau)>=\sigma^{2} \delta(\tau)
$$

Consistency with Eq. (5) is achieved in the data analysis by forming each time series $y(t, \tau)$ with non-overlapping time intervals $\tau$. Defining $D_{0}=<\xi^{2}(t)>/ 2$ we then obtain:

$\gamma(y)=\sqrt{\frac{b_{0}}{D_{0}}} y|y|^{-\frac{1}{2 \alpha}}$,

and

$\beta(y)=\left[b_{0}\left(1-\frac{1}{2 \alpha}\right)-a_{0}\right] y|y|^{-\frac{1}{\alpha}}$.

With $\alpha=1 / 2$ and $a_{0}=0$ one recovers the Brownian random walk with Eq. (18) reduced to a diffusion equation with constant diffusion coefficient.

Interestingly, Beck (2001) has independently proposed a nonlinear Langevin equation where $\beta$ but not $\gamma$ varies with $y$. This yields leptokurtic PDFs of the Tsallis functional form.

Finally the variable $\tau$ in Eq. (18), and $t$ in Eq. (24) can be read in two ways: either as the renormalization variable of the stochastic variable $y(t, \tau)$ or the time variable of $x(t)$ since from Eq. (5) $\tau=2^{n} \Delta$ and with the choice $N=2^{n}$ we have $x(t) \equiv y^{n}(t, \tau), \tau \equiv t$ ( $n, N$ large). Thus (24) can be seen either as a prescription for generating a self-affine time series with scaling exponent $\alpha$, or as describing the renormalization flow.

\section{Conclusions}

Empirical determination of the scaling properties and exponents of time series $x(t)$ presents a formidable challenge in testing, and developing, a theoretical understanding of turbulence and other out-of-equilibrium phenomena. In this paper we have discussed the special case of self affine time series by treating the differenced variable $y(t, \tau)=x(t+\tau)-x(t)$ as increments of a stochastic process (a generalized random walk). We have highlighted two complementary approaches to the data.

The first of these is PDF rescaling; using a low order moment to determine a scaling exponent and then verifying whether this exponent collapses the PDFs of the differenced variable $y(t, \tau)$ over the full range of $y$ accessible from the data. As a corollary this collapsed PDF should also be well described by the solution of a Fokker-Planck equation which has power law transport coefficients.

The second of these is using structure functions to determine the scaling properties of the higher order moments. In a finite length time series the higher order structure functions can be distorted by isolated, extreme events which are not well represented statistically. Using the example of a finite length Lévy flight, we have demonstrated a method for conditioning the time series that can in principle recover the underlying self affine scaling.

Finally, to highlight how both these methods are complementary in quantifying the scaling properties of the time series a fractional Brownian walk was constructed to share the same second moment as an interval of the differenced $A E$ index time series. The two time series were demonstrated to possess very different PDF of the differenced variable, and distinct structure functions.

Both of these approaches could in principle be generalized to multifractal time series (see e.g. Schertzer et al., 2001).

Acknowledgements. BH was supported by the PPARC. We thank J. Greenhough and M. Freeman for stimulating discussions and the UK Solar System Data Centre for the provision of geomagnetic index datasets.

Edited by: A. C. L. Chian

Reviewed by: one referee

\section{References}

Beck, C.: Dynamical foundations of nonextensive statistical mechanics, Phys. Rev. Lett., 87, doi:10.1103/PhysRevLett.87.180601, 2001. 
Chapman, S. C. and Watkins, M. W.: Avalanching and Self Organised Criticality: a paradigm for magnetospheric dynamics?, Space Sci. Rev., 95, 293-307, 2001.

Chechkin, A. V. and Gonchar, V. Yu.: Self-Affinity of Ordinary Lévy Motion, Spurious Multi-Affinity and Pseudo-Gaussian Relations, Chaos, Solitons and Fractals, 11, 2379-2390, 2000.

Consolini, G., Marcucci, M. F., and Candidi, M.: Multifractal structure of auroral electrojet index data, Phys. Rev. Lett., 76, 40824085, 1996.

Consolini, G. and De Michelis, P.: Non-Gaussian distribution function of $A E$ index fluctuations: Evidence for time intermittency, Geophys. Res. Lett., 25, 4087-4090, 1998.

Freeman, M. P., Watkins, N. W., and Riley, D. J.: Evidence for a solar wind origin of the power law burst lifetime distribution of the $A E$ indices, Geophys. Res. Lett.,27, 1087-1090, 2000.

Frisch U.: Turbulence, The legacy of A. N. Kolmogorov, Cambridge University Press, Cambridge, 1995.

Gardiner, C. W.: Handbook of Stochastic Methods: For Physics, Chemistry, and the Natural Sciences (Springer Series in Synergetics), Springer-Verlag, 1986.

Hnat, B., Chapman, S. C., Rowlands, G., Watkins, N. W., and Farrell, W. M.: Finite size scaling in the solar wind magnetic field energy density as seen by WIND, Geophys. Res. Lett., 29, 1446, doi:10.1029/2001GL014587, 2002.

Hnat, B., Chapman, S. C., Rowlands, G., Watkins, N. W., and Freeman, M. P.: Scaling in long term data sets of geomagnetic indices and solar wind $\epsilon$ as seen by WIND spacecraft, Geophys. Res. Lett.,30, 2174, doi:10.1029/2003GL018209 2003a.

Hnat, B., Chapman, S. C., and Rowlands, G.: Intermittency, scaling, and the Fokker-Planck approach to fluctuations of the solar wind bulk plasma parameters as seen by the WIND spacecraft, Phys. Rev. E, 67, 056404-056412, 2003b.

Hnat, B., Chapman, S. C., and Rowlands, G.: Scaling and a FokkerPlanck model for fluctuations in geomagnetic indices and comparison with solar wind epsilon as seen by WIND and ACE, J. Geophys. Res., in press, 2005.

Horbury T. S. and Balogh, A.: Structure function measurements of the intermittent MHD turbulent cascade, Nonlin. Processes Geophys., 4, 185-199, 1997,

\section{SRef-ID: 1607-7946/npg/1997-4-185.}

Kovács, P., Carbone, V., and Vörös, Z.: Wavelet-based filtering of intermittent events from geomagnetic time series, Planetary and Space Science, 49, 1219-1231, 2001.

Mandelbrot, B. B.: Gaussian Self-Affinity and Fractals: Globality, The Earth, $1 / f$ Noise and $R / S$, Springer-Verlag, Berlin, 2002.

Mantegna, R. N. and Stanley, H. E.: Scaling Behavior in the Dynamics of an Economic Index, Nature, 376, 46-49, 1995.

Mangeney, A., Salem, C., Veltri, P. L., and Cecconi, B.: in: Multipoint measurements versus theory, ESA report SP-492, 492, 2001

Nakao, H.: Multiscaling Properties of Truncated Lévy Flights, Phys. Lett. A, 266, 282-289, 2000.

Peters, E. E.: Chaos and Order in the Capital Markets, John Wiley and Sons, New York, 1996.

Schertzer, D., Larcheveque, M., Duan, J., Yanovsky, V. V., and Lovejoy, S.: Fractional Fokker-Planck equation for nonlinear stochastic differentisl equations driven by non-Gaussian Lévy stable noises, J. Math. Phys., 42, 200-212, 2001.

Shlesinger, M. F., Zaslavsky, G. M., and Frisch, U. (eds.): Lévy flights and related topics in physics: proc. int. workshop Nice, France, 27-30 June, 1994, Lecture Notes in Physics: 450, Springer-Verlag, Berlin, 1995.
Siegert, S. and Friedrich, R.: Modeling of Lévy processes by data analysis, Phys. Rev. E, 64, doi:10.1103/PhysRevE.64.041107, 2001.

Sornette, D.: Critical Phenomena in Natural Sciences; Chaos, Fractals, Self-organization and Disorder: Concepts and Tools, Springer-Verlag, Berlin, 2000.

Sorriso-Valvo, L., Carbone, V., Giuliani, P., Veltri, P., Bruno, R., Antoni, V., and Martines, E.: Intermittency in plasma turbulence, Planet. Space Sci., 49, 1193-1200, 2001.

Stepanova, M. V., Antonova, E. E., and Troshichev, O.: Intermittency of magnetospheric dynamics through non-Gaussian distribution function of PC-index fluctuations, Geophys. Res. Lett., 20 30, 1127, doi:10.1029/2002GL016070, 2003.

Takalo, J., Timonen, J., and Koskinen, H.: Correlation dimension and affinity of $A E$ data and bicolored noise, Geophys. Res. Lett., 20, 1527-1530, 1993.

Takalo, J. and Timonen, J.: Comparison of the dynamics of the $A U$ and $P C$ indices, Geophys. Res. Lett., 25, 2101-2104, 1998.

Tsurutani, B. T., Smith, E. J., Buti, B., Matsumoto, H., Brinca, A.: The nonlinear response of $A E$ to the IMF $B_{S}$ driver: A spectral break at 5 hours, Geophys. Res. Lett., 17, 1870-1820, 1990.

Tu, C. -Y. and Marsch, E.: MHD Structures, waves and turbulence in the solar wind: Observations and theories, Space Sci. Rev. 73, $1-2,1-210,1995$.

Uritsky V. M. and Pudovkin, M. I.: Low frequency 1/f-like fluctuations of the $A E$ index as a possible manifestation of selforganized criticality in the magnetosphere, Ann. Geophys., 16, 12, 1580-1588, 1998,

SRef-ID: 1432-0576/ag/1998-16-1580.

Uritsky, V. M., Klimas, A. J., and Vassiliadis, D.: Comparative study of dynamical critical scaling in the auroral electrojet index versus solar wind fluctuations, Geophys. Res. Lett., 28, 38093812, 2001.

Vörös, Z., Kovács, P., Juhász, Á., Körmendi, A., and Green, A. W.: Scaling laws from geomagnetic time series, J. Geophys. Res., 25, 2621-2624, 1998.

Vörös, Z., Jankovičová, D., and Kovács, P.: Scaling and singularity characteristics of solar wind and magnetospheric fluctuations, Nonlin. Processes Geophys., 9, 2, 149-162, 2002,

SRef-ID: 1607-7946/npg/2002-9-149.

Watkins, N. W., Freeman, M. P., Rhodes, C. S., and Rowlands, G.: Ambiguities in determination of self-affinity in the $A E$ index time series, Fractals, 9, 471-479, 2001.

Weigel, R. S. and Baker, D. N.: Probability distribution invariance of 1-minute auroral-zone geomagnetic field fluctuations, Geophys. Res. Lett., 20 30, 23, 2193, doi:10.1029/2003GL018470, 2003a.

Wilson, K. G.: Problems in physics with many scales of length, Scientific American, 241, 140, 158-179, 1979.

Yannacopoulos, A. N. and Rowlands, G.: Local transport coefficients for chaotic systems, J. Phys. A Math. Gen. 30, 1503-1525, 1997.

Zaslavsky, G. M.: From Lévy flights to the Fractional Kinetic Equation for dynamical chaos, 216, p. in: Lévy flights and related topics in physics: proc. int. workshop Nice, France, 27-30 June, 1994, edited by: Shlesinger, M. F., Zaslavsky, G. M., and Frisch, J., Lecture Notes in Physics, 450, Springer-Verlag, Berlin, 1995. 